\title{
SPIRAL2 : une sonde de nouvelle génération pour explorer la matière nucléaire
}

Alain Savalle (alain.savalle@ganil.fr), Pascal Jardin, Robin Ferdinand, Xavier Ledoux, Jean-Charles Thomas, Hervé Savajols et Patrick Bertrand

Grand accélérateur national d'ions lourds (GANIL), CNRS/CEA, BP 55027, 14076 Caen Cedex 5

SPIRAL2 est un accélérateur d'ions de nouvelle génération, qui va agrandir et renforcer le GANIL (Grand accélérateur national d'ions lourds, installé à Caen et géré par le CEA et le CNRS).

Il fournira des faisceaux d'ions lourds et légers d'intensité inégalée, qui seront utilisés pour produire de nouveaux éléments superlourds et compléter ainsi le tableau de Mendeleïev, pour créer et étudier des noyaux exotiques qui n'existent que dans le cosmos, ou encore pour étudier les effets de l'irradiation de la matière par les neutrons rapides.

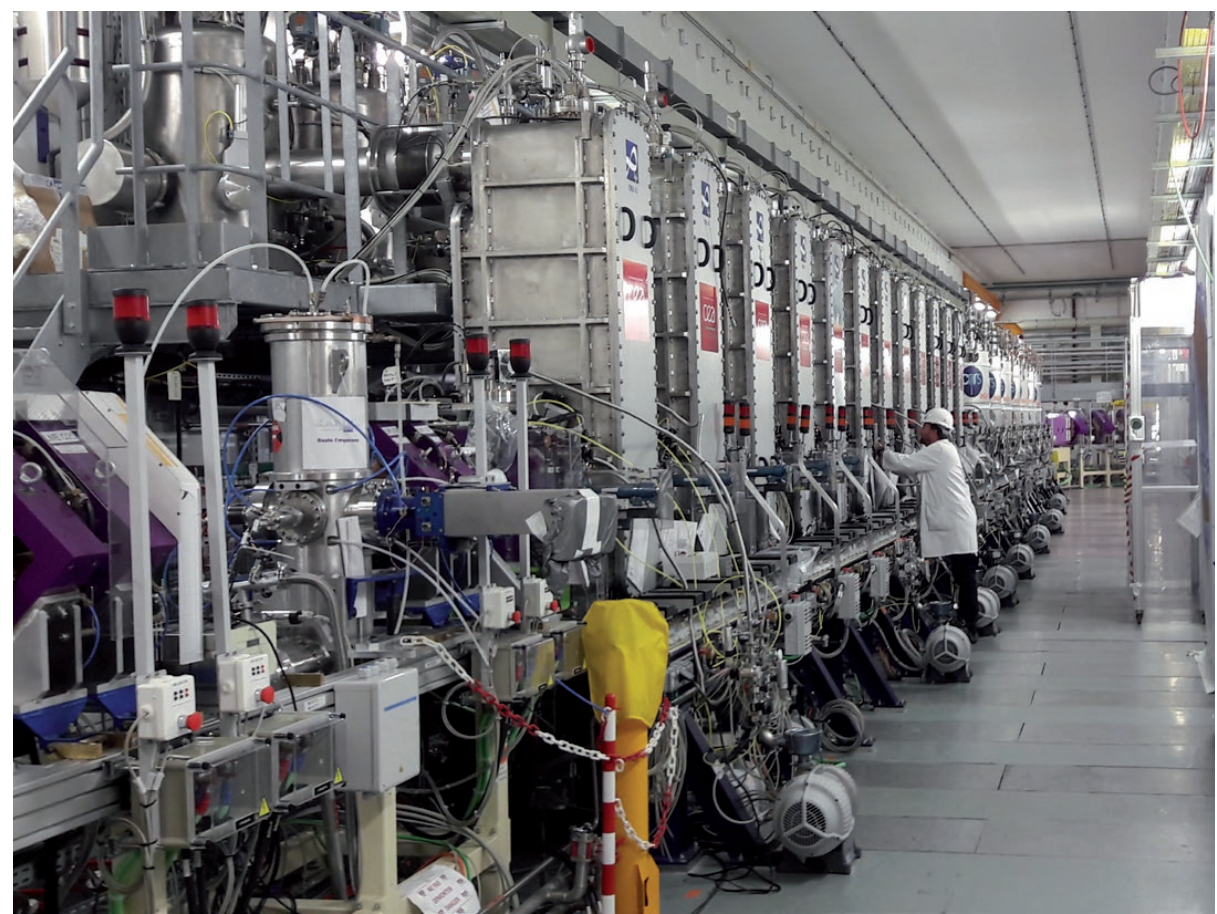

Vue de l'accélérateur linéaire (LINAC) de SPIRAL2.

Le projet SPIRAL2 (Système de Production d'Ions Radioactifs Accélérés en Ligne de $2^{\mathrm{e}}$ génération) est en cours d'achèvement sur le site du Grand Accélérateur National d'Ions Lourds (GANIL) situé à Caen.

Ce projet, lancé en 2005, a couté environ $165 \mathrm{M} €$ (main d'œuvre incluse), financés par les tutelles du GANIL (CEA et CNRS/IN2P3) et les collectivités locales, et est issu de nombreuses collaborations avec des laboratoires français, européens et internationaux. Il consiste en deux sources d'ions, un accélérateur linéaire supracon- ducteur qui produira des faisceaux d'ions d'une intensité exceptionnelle et (dans un premier temps) deux salles d'expériences, qui permettront de poursuivre l'étude du noyau atomique et des forces fondamentales qui en déterminent les propriétés.

La construction du bâtiment a débuté en 2011 et s'est achevée en 2014. SPIRAL2 a été inauguré le 3 novembre 2016 par le président François Hollande. Les premières expériences de physique sont prévues en 2020. 


\section{I - Le projet scientifique}

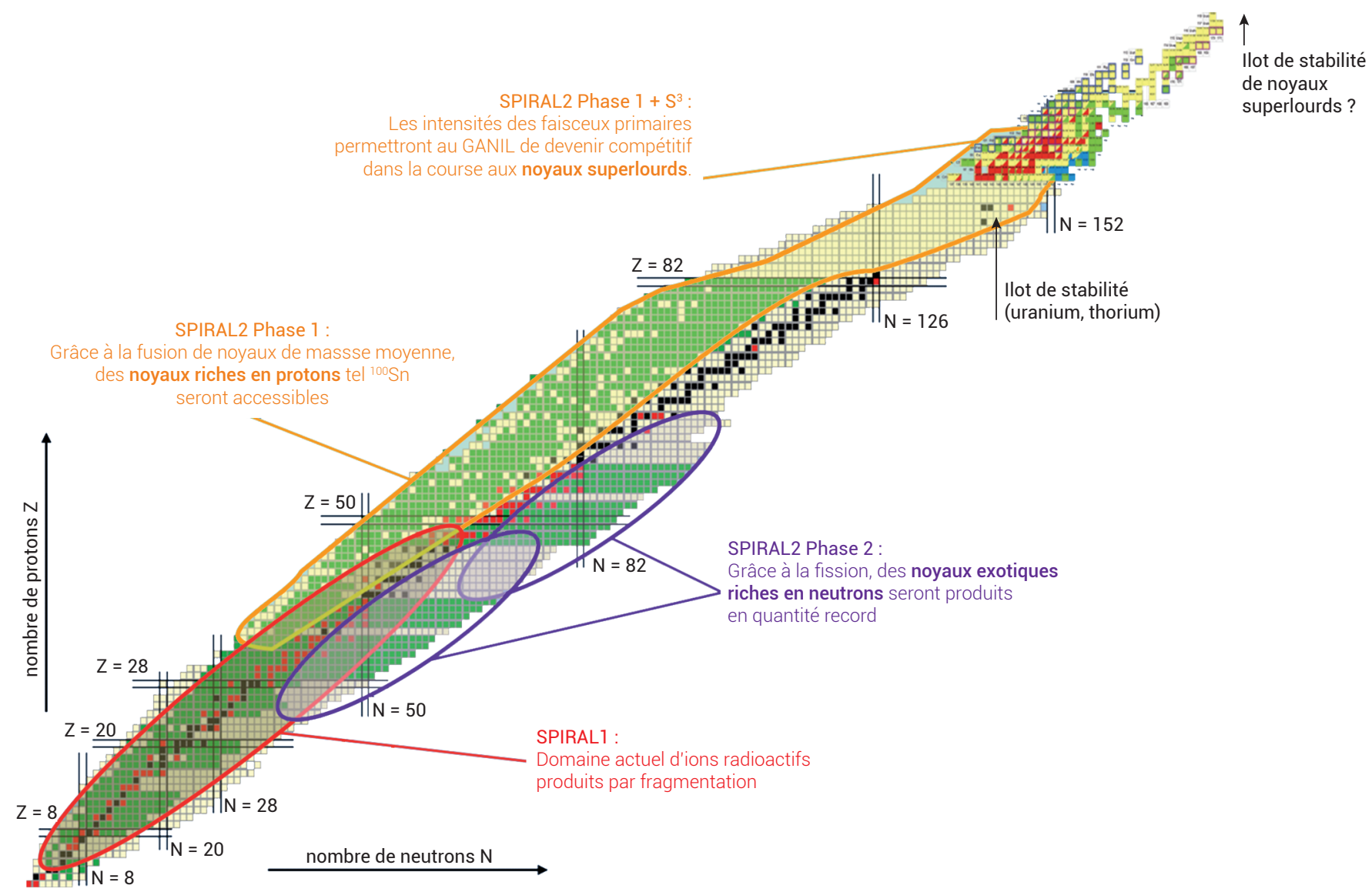

1. Carte des noyaux en fonction de leurs nombres de protons $(\mathrm{Z})$ et de neutrons $(\mathrm{N})$, avec localisation des régions de noyaux radioactifs qui seront accessibles avec la future installation SPIRAL2 au GANIL. Les noyaux existant à l'état naturel, situés au voisinage de la " vallée de stabilité ", sont représentés en rouge (s'ils sont accélérés au GANIL) ou en noir. Les noyaux exotiques produits ou qui seront produits au GANIL sont indiqués en vert. Pour comparaison, la région de noyaux radioactifs produits avec les installations actuelles du GANIL, dont SPIRAL1, est reportée.

$>>$

$\mathrm{Au}$ GANIL, l'exploration de la matière nucléaire a commencé en 1983. De très nombreux résultats obtenus depuis cette date ont fait progresser nos connaissances, mais il reste encore de grandes questions sur l'origine et les propriétés de la matière. - Où et comment sont produits les atomes qui constituent notre monde ? Après le Big Bang originel, survenu il y a 13,8 milliards d'années, la matière alors présente s'est refroidie pour donner en germe les particules constituant les noyaux atomiques. Pour comprendre leur (nucléo)synthèse ainsi que leur abondance relative, il est nécessaire d'étudier les processus qui, dans les étoiles, permettent de produire des noyaux atomiques. Cette compréhension passe par l'étude de noyaux de synthèse, produits par collisions violentes entre noyaux stables, seuls disponibles sur Terre.
- Lors de la collision des faisceaux d'ions avec une cible, quelles informations pouvons-nous obtenir sur la forme des noyaux produits, l'organisation de leurs nucléons, ainsi que sur les interactions entre protons et neutrons au cœur de l'atome?

- D'où provient l'existence des noyaux "magiques ", particulièrement robustes lorsqu'ils possèdent certains nombres de neutrons ou de protons, et comment évoluent-ils lorsque les noyaux comptent un très grand excès des uns ou des autres?

- Comment former des éléments encore plus lourds que l'oganesson, noyau contenant le plus grand nombre de protons (118) observé à ce jour ? Peut-on former des noyaux "superlourds ", comportant plus de 120 protons?
Ces recherches passent par la production et l'observation de noyaux dits " exotiques", puisqu'absents naturellement de notre monde. Cette exploration, entamée il y a moins d'un siècle (Rutherford, Cockroft et Walton...), a déjà permis d'étudier près de trois mille noyaux exotiques (sans pour autant pouvoir tous les caractériser finement), alors que la théorie prédit l'existence de cinq à sept mille noyaux (fig. 1).

Regardons la figure 1. Sur une ligne de nombre atomique $\mathrm{Z}$ donné (échelle verticale), lorsque partant de ces noyaux stables l'on parcourt l'échelle horizontale en diminuant le nombre de neutrons (" noyaux riches en protons ") ou en augmentant ce nombre (" noyaux riches en neutrons"), on obtient un noyau de plus en plus " exotique ». À mesure que l'on s'éloigne de la "vallée de stabilité ", produire un noyau devient de plus en plus difficile, et 
sa durée de vie diminue rapidement jusqu'à ce que soient atteintes les "limites d'existence " de la matière nucléaire. L'installation SPIRAL2 va permettre d'atteindre des régions de la carte des noyaux peu explorées jusqu'ici, en particulier celle des "noyaux superlourds " tout en haut de la carte.

L'attention des physiciens nucléaires s'est tout d'abord portée sur les noyaux exotiques légers, plus simples à produire du fait de la proximité des limites d'existence des noyaux. Pour permettre aux physiciens de progresser vers des régions plus exotiques encore, et faire de ces noyaux exotiques de nouveaux projectiles, des installations dédiées à la production de faisceaux d'ions exotiques ont été construites [1] (ISOLDE au CERN en Suisse, TRIUMF au Canada, puis SPIRAL1 au GANIL au début des années 2000).

L'installation SPIRAL2 s'inscrit dans cette démarche. Son but est de disposer au sein du GANIL d'une association unique d'instruments permettant d'étendre la palette de noyaux exotiques produits et assemblés en faisceaux de grande qualité, de manière à permettre leur étude avec une grande précision. En complément, d'autres expériences de physique nucléaire pourront se faire également avec des faisceaux de neutrons intenses et collimatés pour induire des réactions nucléaires, dont la fission.

En plus des recherches en physique nucléaire, SPIRAL2 permettra aussi des recherches en physique atomique, en physique des matériaux et en radiobiologie.

La première phase du projet a consisté en la construction d'un accélérateur linéaire et de deux salles d'expériences : NFS (Neutrons For Science) et $S^{3}$ (Super Séparateur Spectromètre). L'accélérateur linéaire de SPIRAL2 sera capable de produire une grande variété de faisceaux d'ions d'isotopes stables, allant du proton $(Z=1)$ jusqu'au nickel $(Z=28)$. Les faisceaux d'ions légers (protons, deutons), qui ne sont pas disponibles actuellement au GANIL, serviront surtout à produire des faisceaux de neutrons alimentant la salle NFS. Les faisceaux d'ions " lourds " (depuis l'hélium jusqu'au nickel dans la première phase) généreront, par interaction avec une cible, des faisceaux d'ions radioactifs d'une intensité exceptionnelle (10 à 100 fois plus que celles des faisceaux produits par le GANIL actuel) qui seront utilisés dans la salle $S^{3}$.

Cette phase 1 sera complétée à partir de 2023 par la plateforme DESIR (Désintégration, Excitation et Stockage d'Ions Radioactifs) puis, plus tard, par le futur injecteur $Q / A=1 / 7$ ( $Q$ : charge de l'ion, $A=Z+N$ : nombre de nucléons), qui permettra d'accélérer jusqu'à l'uranium 238. Enfin, une seconde phase du projet prévoit la production et l'étude de faisceaux d'ions radioactifs produits par fission d'une cible de carbure d'uranium, ces faisceaux étant post-accélérés et étudiés dans l'installation GANIL originelle. À terme, les deux installations GANIL et SPIRAL2 n'en feront alors plus qu'une, comme illustré par la figure 2 .

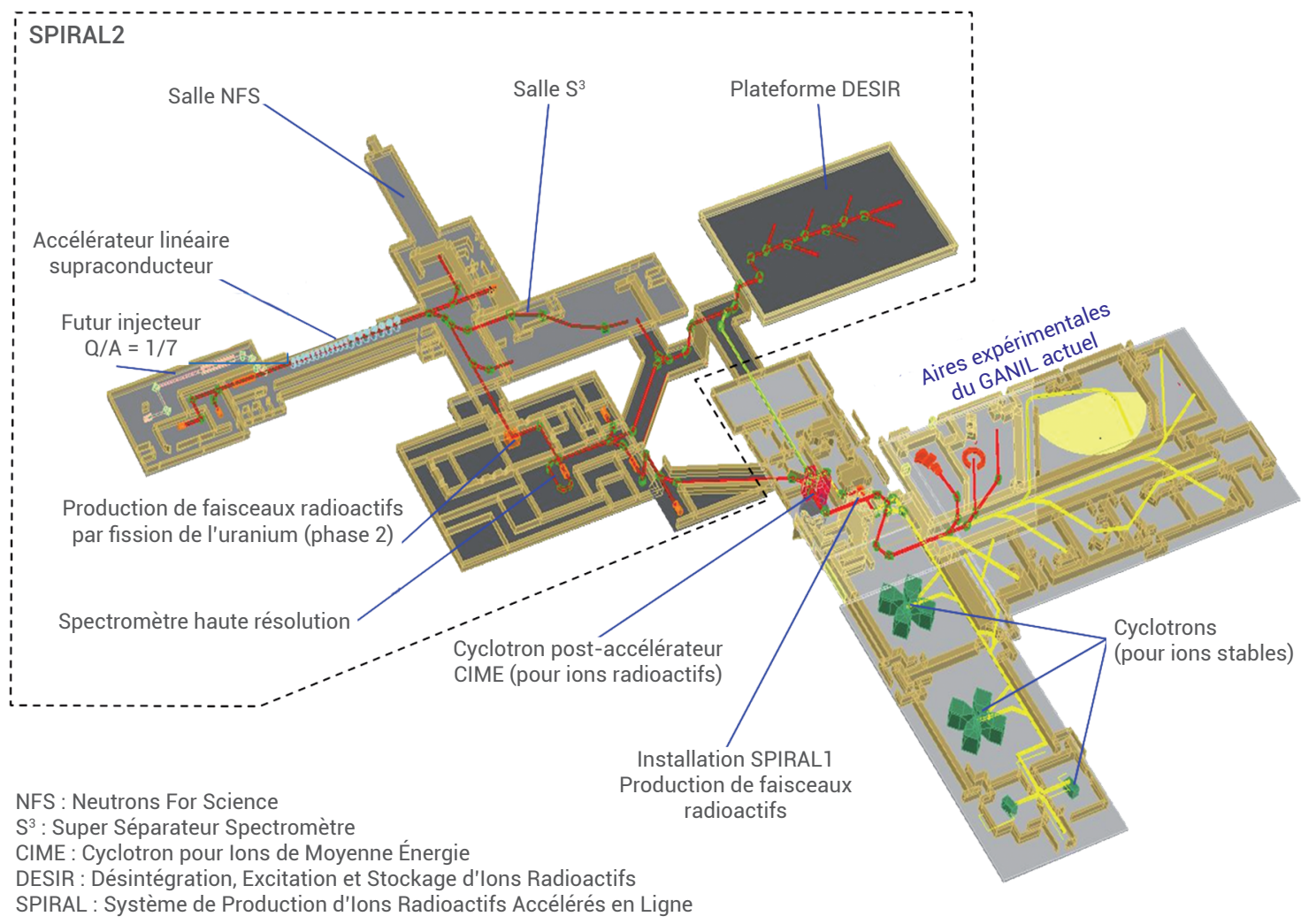

2. Schéma de l'installation du GANIL après construction de SPIRAL2, incluant la phase $\mathbf{2}$ (bâtiment de production de faisceaux radioactifs, par fission de l'uranium, dont le calendrier de construction n'est pas encore arrêté). La surface totale du GANIL sera alors presque doublée (de 11000 à environ $20000 \mathrm{~m}^{2}$ ). Sont représentés en rouge les chemins possibles des faisceaux de SPIRAL2, et les chemins possibles pour les faisceaux actuels du GANIL. 


\section{II - L'accélérateur}

$>>$

Le nouvel accélérateur linéaire de SPIRAL2 est le fruit d'une collaboration entre plusieurs laboratoires français et étrangers [2]. L'une des principales caractéristiques de l'ensemble accélérateur est qu'il doit être capable d'accélérer des faisceaux d'ions légers (protons $\mathrm{H}^{+}$et deutons $\mathrm{D}^{+}$) tout autant que des ions lourds, dans des gammes d'énergie et d'intensité très larges, comme illustré dans le tableau 1.

Un schéma de principe de l'accélérateur est donné dans la figure 3. Deux sources d'ions permettent la production, soit de faisceaux d'ions légers (protons, deutons constitués d'un proton et d'un neutron), soit de faisceaux d'ions "lourds " (de l'hélium jusqu'au nickel). Le premier étage d'accélération est constitué d'un quadripôle radiofréquence (RFQ), dont le rôle est de grouper les ions en paquets à la fréquence d'accélération et de réaliser une première accélération jusqu'à l'énergie permettant l'injection dans l'accélérateur suivant. Celui-ci, l'accélérateur linéaire (abrégé LINAC en anglais), est constitué de 26 cavités accélératrices élaborées par des laboratoires du CEA et du CNRS. Elles sont supraconductrices.

Les ions légers sont produits par une source développée par l'IRFU/Saclay, qui permet de délivrer un courant de $5 \mathrm{~mA}$ d'ions $\mathrm{H}^{+}$ou $\mathrm{D}^{+}$de façon très stable. Pour permettre d'accélérer les ions lourds en utilisant le même RFQ, la source d'ions lourds, construite par le laboratoire LPSC/ Grenoble, doit produire des ions de rapport charge-sur-masse inhabituellement élevé pour ce type de source (i.e. Q/A 1/3 jusqu'à $A \sim 60)$, tout en délivrant des intensités sans précédent.

Le RFQ est une cavité en cuivre massif de $5 \mathrm{~m}$ de long. Les faisceaux d'ions passent au centre de quatre pôles électriques (fig. 4). L'oscillation à haute fréquence (88 MHz) de la cavité, associée à des ondulations sur l'extrémité des pôles, génère un potentiel électrique qui permet de focaliser le faisceau d'ions, le regrouper en paquets à la fréquence de la machine, et de l'accélérer lors de sa progression dans la cavité. L'énergie de sortie des ions est de 0,75 MeV/A (ce qui correspond, quel que soit $A$, à $4 \%$ de la vitesse de la lumière).

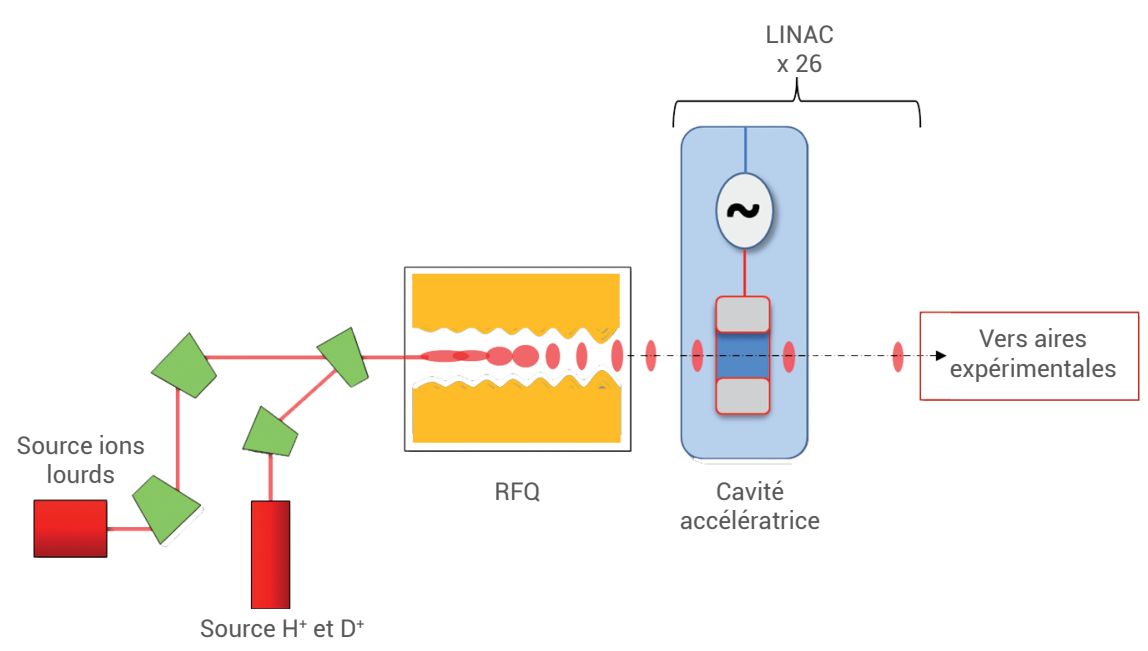

3. Constitution de l'ensemble de production et d'accélération de SPIRAL2. Les faisceaux continus issus des deux sources d'ions sont mis en paquets par un quadripôle radiofréquence (RFQ), puis accélérés par le même accélérateur linéaire constitué de 26 cavités accélératrices en cascade. Les trapèzes en vert correspondent aux dipôles magnétiques qui permettent d'effectuer la sélection des ions en fonction de leur rapport charge-sur-masse.

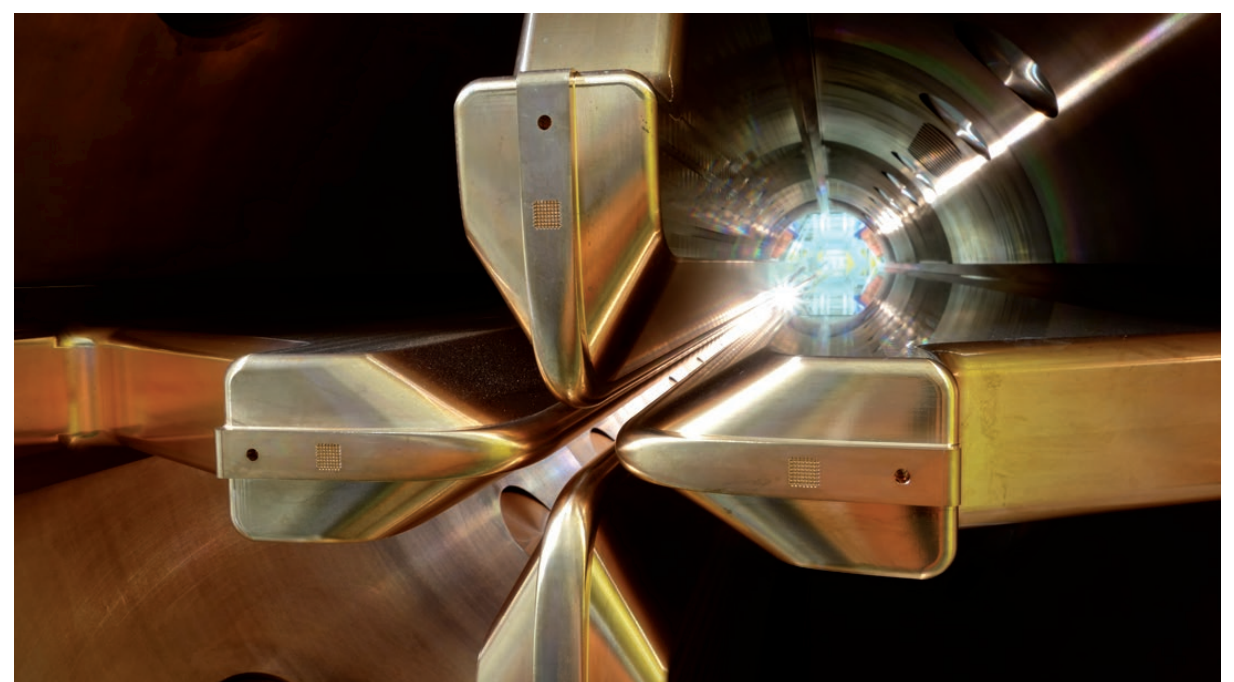

4. Vue interne du quadripôle radiofréquence (RFQ) de SPIRAL2 : le faisceau d'ions passe au centre des quatre pôles.

\begin{tabular}{|c|c|c|c|c|}
\hline Faisceau & $\mathrm{H}^{+}$ & $\mathrm{D}^{+}$ & $\begin{array}{c}\text { lons } \\
\text { lourds }\end{array}$ & $\begin{array}{c}\text { Ions lourds } \\
\text { (projet) }\end{array}$ \\
\hline Rapport charge/masse (Q/A) & 1 & $1 / 2$ & $1 / 3$ & $1 / 6$ à $7 / 7$ \\
\hline Intensité maximum (mA) & 5 & 5 & 1 & 1 \\
\hline Énergie minimum (MeV/A) & 2 & 2 & 2 & 2 \\
Énergie maximum E (MeV/A) & 33 & 20 & 14,5 & 8 \\
\hline Puissance maximum du faisceau (kW) & 165 & 200 & 44 & 48 \\
\hline
\end{tabular}

Tableau 1. Principales caractéristiques des faisceaux qui seront accélérés dans le cadre du projet SPIRAL2. La production et l'accélération d'ions lourds de rapport charge-sur-masse égal à 1/6 ou $1 / 7$ est en projet. 
Les lignes de transport du faisceau, depuis les sources d'ions jusqu'aux salles d'expériences, sont constituées d'éléments magnétiques (dipôles, quadripôles, hexapôles...) destinés à guider et mettre en forme le faisceau, et de nombreux diagnostics qui permettent de s'assurer de sa bonne transmission (mesures de position, d'intensité, de phase...).

Dans l'accélérateur linéaire, les ions préalablement rassemblés en paquets grâce au RFQ subissent des accélérations successives lors de leur passage dans des cavités. À la différence des cyclotrons qui enroulent les ions en spirale grâce à un champ magnétique pour leur permettre de passer plusieurs fois dans la même cavité accélératrice, les accélérateurs linéaires sont constitués d'une succession de cavités accélératrices dans lesquelles les ions ne passent qu'une seule fois. Si les cyclotrons ont donc généralement l'avantage d'être plus compacts et moins onéreux que les accélérateurs linéaires, ces derniers ont cependant un avantage déterminant sur les cyclotrons : l'intensité du faisceau d'ions accéléré n'est pas limitée. Les intensités requises par les objectifs scientifiques de SPIRAL2 n'étaient pas compatibles avec les pertes inévitables à l'éjection d'un cyclotron.

Le LINAC (photo p. 11) est à la pointe de la technique mondiale. Il est le fruit de nombreuses études et optimisations. Il fonctionne en mode supraconducteur, ce qui permet aux cavités de produire un champ électrique accélérateur beaucoup plus important qu'une ancienne technologie non supraconductrice, tout en nécessitant une puissance d'onde à haute fréquence beaucoup plus faible. L'économie associée et les compétences des laboratoires partenaires du GANIL, spécialisés dans ce domaine, ont naturellement conduit le projet SPIRAL2 à opter pour cette technologie.

Le choix des cavités supraconductrices s'est porté sur un ensemble de cavités " quart d'ondes " (QWR en anglais), fonctionnant toutes à $88 \mathrm{MHz}$ et disposées dans des cryostats assez courts. L'ensemble constitué des cavités et du cryostat est appelé cryomodule. Ces cavités, fabriquées en niobium massif ultrapur, fonctionnent de façon optimale à une température absolue d'environ 4,5 K. Elles sont maintenues à cette température extrême grâce à une " usine cryogénique » assurant une circulation d'hélium liquide. Les cavités permettent d'atteindre des champs électriques de $6,5 \mathrm{MV} / \mathrm{m}$. Les ions ainsi accélérés pourront atteindre jusqu'à $26 \%$ de la vitesse de la lumière, selon les besoins de la physique.

Entre chaque cryomodule, une section fonctionnant à température ambiante permet la conduite et le diagnostic du faisceau.

L'accélérateur est soumis à un ensemble d'exigences réglementaires en termes de sécurité et de sûreté, similaires aux exigences de conception des centrales nucléaires : protection de l'environnement vis-à-vis des rejets et des rayonnements, pérennité des infrastructures en cas d'agression extérieure (chute d'aéronef ou séisme par exemple), limitation des risques pour les travailleurs, etc. Le respect de ces exigences a eu des conséquences très importantes lors de la construction : du bâtiment simple requis pour abriter le procédé, il a été nécessaire de passer à la construction d'un bâtiment "nucléarisé ", dont les murs remplissent des fonctions de protection biologique, de résistance au feu, de tenue au séisme et de confinement.

\section{III - Les expériences}

\section{La salle NFS : étudier les neutrons rapides et leur interaction avec la matière}

Les réactions induites par les neutrons sur les noyaux des atomes jouent un rôle très important en recherche fondamentale et sont utilisées dans de nombreuses applications : fonctionnement des réacteurs nucléaires, production d'énergie par fusion, tests de résistance de composants électroniques aux radiations, production de radioéléments à usage médical, etc. Pour développer ces applications, les physiciens et ingénieurs emploient des codes de simulation utilisant des bases de données nucléaires. Les «sections efficaces » en font partie : ces informations, très importantes, indiquent la probabilité, pour chaque noyau d'atome, qu'une réaction avec une particule incidente d'énergie donnée se produise. La validité des simulations dépend en partie de la qualité de ces données nucléaires. Dans de nombreux cas - réactions de fission, capture d'un neutron, production de particules légères (protons, noyaux d'hélium, neutrons, photons $\gamma$ ), etc. - les sections efficaces sont inconnues, ou connues avec une précision insuffisante. Il existe notamment une forte demande de données pour les réactions induites par neutrons entre quelques $\mathrm{MeV}$ et $40 \mathrm{MeV}$, un domaine d'énergie que peu d'installations permettent de couvrir. C'est pourquoi le projet Neutrons for Science (NFS) a été initié [3]. Il s'agit de fournir aux expérimentateurs un outil permettant d'étudier les réactions induites sur des noyaux d'atomes par des neutrons de haute énergie (allant jusqu'à $40 \mathrm{MeV}$ ), avec des flux inégalés, une résolution en énergie et un rapport signal/bruit optimisés pour les expériences de physique nucléaire de grande précision.
NFS est composé de deux salles séparées par un épais mur de béton, dans lequel s'insère un collimateur (fig. 5). Les neutrons sont produits dans la première salle, dite « salle du convertisseur ». Une partie de ces neutrons traverse le collimateur, produisant ainsi un faisceau disponible dans la seconde salle, dite "salle de temps de vol ".

Les neutrons sont générés par l'interaction des faisceaux de protons ou de deutons accélérés par le LINAC avec un convertisseur. Un faisceau de deutons interagissant avec un convertisseur épais (8 $\mathrm{mm}$ ) en béryllium ou en carbone permet de produire des neutrons avec un spectre en énergie continu, s'étendant de $100 \mathrm{keV}$ à $40 \mathrm{MeV}$, et un flux disponible de $5.10^{11} \mathrm{n} / \mathrm{cm}^{2} / \mathrm{s}$ en aval du convertisseur et de $510^{7} \mathrm{n} / \mathrm{cm}^{2} / \mathrm{s}$ dans la salle de temps de vol, soit près d'un facteur 100 supérieur aux installations existantes entre 10 et $20 \mathrm{MeV}$. L'utilisation d'un faisceau de protons et d'un convertisseur mince $(1 \mathrm{~mm})$ en lithium permet 
\〉

quant à elle de produire des neutrons avec un spectre quasi monoénergétique. Dans ce cas, l'aimant en aval du convertisseur dévie vers un arrêt de faisceau les protons n'ayant pas interagi avec celui-ci.

La salle de temps de vol recevra des dispositifs permettant de réaliser des expériences avec une très bonne résolution en énergie (de l'ordre de 1\% pour des neutrons de $40 \mathrm{MeV}$ ). La cible, placée dans le faisceau de neutrons, sera entourée de détecteurs permettant d'observer les particules et rayonnements générés au cours de la réaction. Un soin particulier a été apporté à la conception de cette salle, afin de minimiser les sources de bruit de fond (neutrons et photons gamma).

Les flux élevés et la possibilité d'utiliser des cibles d'actinides (famille d'atomes dont les noyaux comptent entre 89 et 103 protons, et dont fait partie l'uranium) rendront possibles certaines expériences jusqu'alors irréalisables dans des temps raisonnables. L'installation sera également utilisée pour irradier des composants électroniques ou des cellules biologiques.

\section{La salle $S^{3}$ : produire et étudier les éléments superlourds}

Un des défis actuels de la physique nucléaire est de produire de nouveaux noyaux situés au-delà des limites connues et d'étudier leurs propriétés. Dans cette optique, la synthèse de nouveaux éléments dits superlourds, avec un nombre de protons toujours plus élevé ( $\mathrm{Z}=114,115$, $116,117,118)$, est motivée par la prédiction de l'existence d'un îlot de stabilité (en haut et à droite de la figure 1) pour des noyaux de masse extrême [4], îlot dont les limites et les dimensions sont aujourd'hui incertaines. L'existence de ces noyaux superlourds, en principe compromise par l'intense répulsion coulombienne entre protons, s'explique par l'organisation en couches des nucléons. Cette structure en couches, fournissant une stabilité fortement accrue, est observée sur des noyaux plus légers possédant des nombres dits " magiques " de nucléons $(8,28,50,82)$. Le nombre magique suivant est 126 pour les neutrons (ce qui a été vérifié expérimentalement); mais sa valeur est incertaine pour les protons, car les modèles théoriques actuels le placent à 114, 120 ou 126 selon les hypothèses utilisées. Les noyaux super-

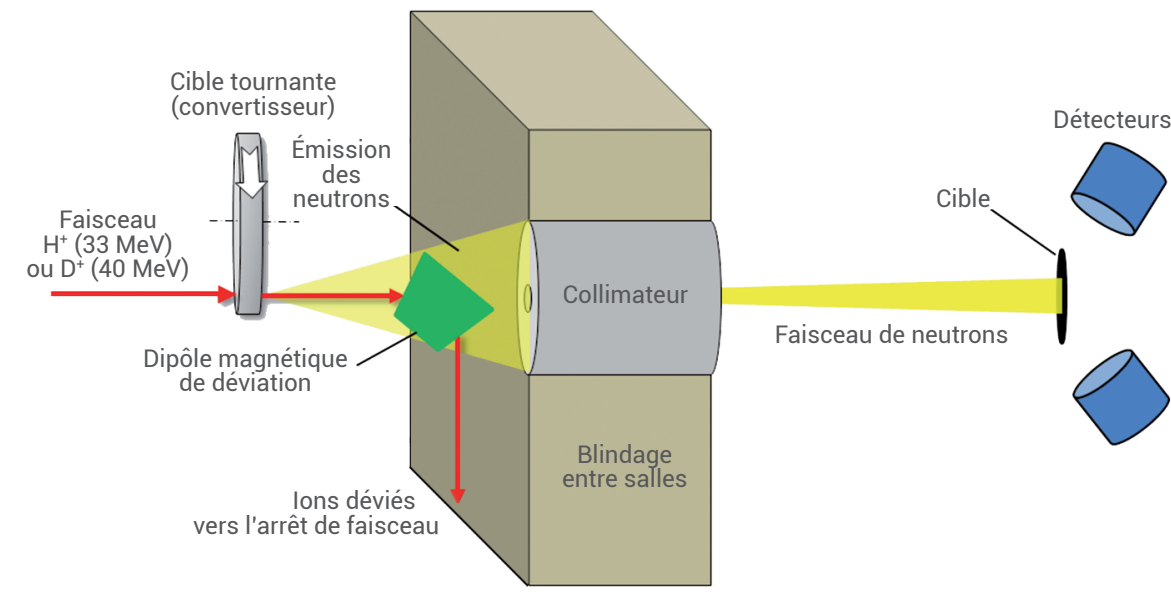

Salle de production du faisceau de neutrons (Salle du convertisseur)

Salle d'expériences (Salle de temps de vol)

5. Principe du dispositif NFS de production des faisceaux de neutrons. Le spectre en énergie des neutrons dépend de la nature et de l'épaisseur du convertisseur. Un faisceau mono-énergétique requiert un convertisseur mince. Dans ce cas, les ions incidents traversent le convertisseur, puis sont déviés à l'aide d'un dipôle magnétique. Les neutrons émis dans l'axe du faisceau d'ions incident poursuivent leur course à travers un collimateur qui, associé au blindage entre salles, contribue à la réduction du bruit de fond dans la salle d'expérience où sont réalisées les mesures. lourds doivent donc être produits en laboratoire, afin de vérifier la position de cet îlot de stabilité et d'observer leurs propriétés.

Cet îlot serait donc un excellent « laboratoire " pour étudier le comportement de la structure nucléaire aux limites de nos connaissances. Des résultats préliminaires montrent que pour les noyaux de cette région, les homologies physico-chimiques qui donnent sa cohérence à la table de Mendeleiev pourraient disparaître, ou être décalées. Cela apporterait des informations importantes pour notre compréhension des lois physiques et chimiques des systèmes atomiques.
Le spectromètre $S^{3}$ a été développé afin d'utiliser des faisceaux d'ions de très haute intensité du LINAC pour produire et sélectionner ces noyaux très exotiques. Ses deux étages optiques, constitués de dipôles magnétiques et électriques (fig. 6), assurent la réjection du faisceau incident et la sélection en masse des noyaux transmis. $\mathrm{S}^{3}$ est dit de nouvelle génération, car il utilise la technologie supraconductrice pour les éléments de transport du faisceau.

La production des noyaux est assurée par l'envoi d'un faisceau d'ions lourds sur une cible rotative pouvant supporter les faisceaux de très haute puissance, et constituée de

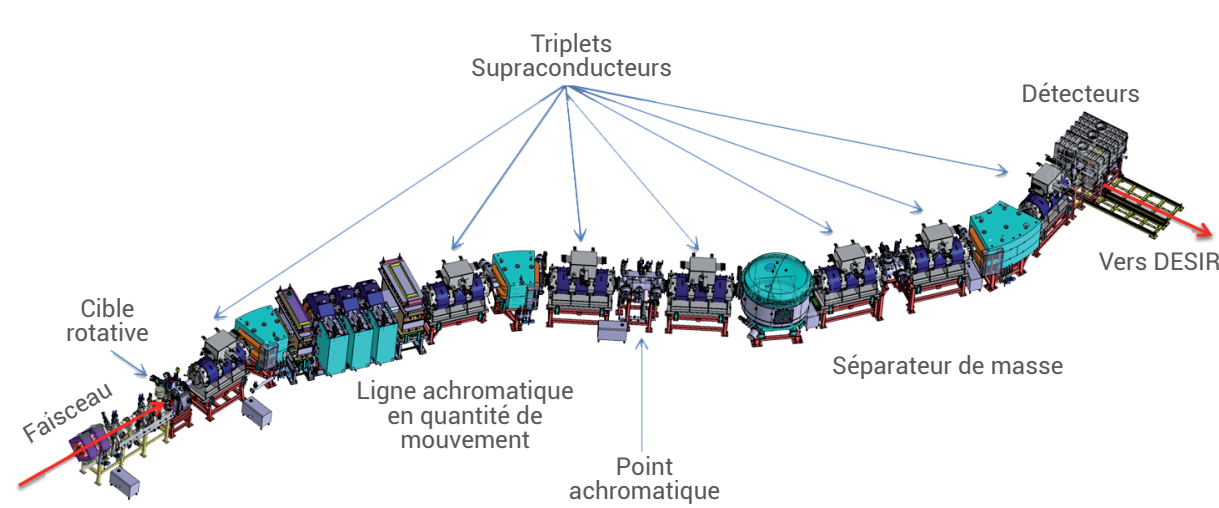

6. Le Super Séparateur Spectromètre $S^{3}$. Les détecteurs SIRIUS et REGLIS seront installés dans le plan focal du spectromètre ("Détecteurs »). L'expérience FISIC prendra place au point achromatique intermédiaire. Le dispositif REGLIS fournira aussi des faisceaux d'ions à la plate-forme DESIR (voir p. 17). 
matériaux radioactifs (actinides) pour les expériences spécifiques d'étude et de synthèse d'éléments superlourds. Au plan focal de $S^{3}$, deux expériences sont prévues pour étudier les propriétés nucléaires et atomiques de ces éléments : SIRIUS et REGLIS3. SIRIUS est un instrument de spectroscopie à haute résolution, qui utilisera les émissions radioactives (particules alpha, rayons gamma et X...) des noyaux superlourds produits et sélectionnés dans $\mathrm{S}^{3}$ pour les identifier et découvrir l'organisation spécifique de leurs nucléons. REGLIS utilise la capacité de certains lasers à ioniser sélectivement les atomes pour identifier et obtenir des informations sur la taille de leur noyau et l'organisation de leurs nucléons. Cette même installation produira aussi, par ionisation laser, les faisceaux d'ions qui seront transmis à l'installation DESIR (voir ci-dessous).

En physique atomique, les modifications transitoires du cortège électronique d'ions traversant la matière sont la clé de voûte de la compréhension de l'endommagement des matériaux soumis à une irradiation et des transferts d'énergie dans les plasmas. Aujourd'hui, ces effets sont mal connus aux énergies cinétiques où ces ions lourds rapides déposent le maximum de leur énergie. L'investigation des collisions ionion n'en est qu'à ses balbutiements, alors que ces interactions sont parmi les phénomènes les plus répandus dans l'Univers et constituent l'un des verrous pour la compréhension de la fusion inertielle ou pour la caractérisation des plasmas stellaires et interstellaires. Le programme d'expériences du projet FISIC ("Fast Ion-Slow Ion Collision"), qui sera implanté dans la salle $\mathrm{S}^{3}$, a pour ambition de distinguer clairement la contribution des différents processus électroniques conduisant à un changement de la charge des ions, incluant une détermination des effets de corrélations entre électrons du cortège atomique. Dans un premier temps, l'étude portera sur un système à trois corps, consistant en un ion possédant un seul électron et un autre ion complètement épluché de ses électrons. Cet ensemble simple permettra un test drastique des théories existantes. Au-delà, le rôle d'électrons additionnels - ajoutés un par un - sera exploré pour une très grande variété de systèmes. En maitrisant l'état électronique des deux partenaires de la collision, l'ensemble des expériences fournira des données inédites sur la dynamique quantique des systèmes à $\mathrm{N}$-corps.

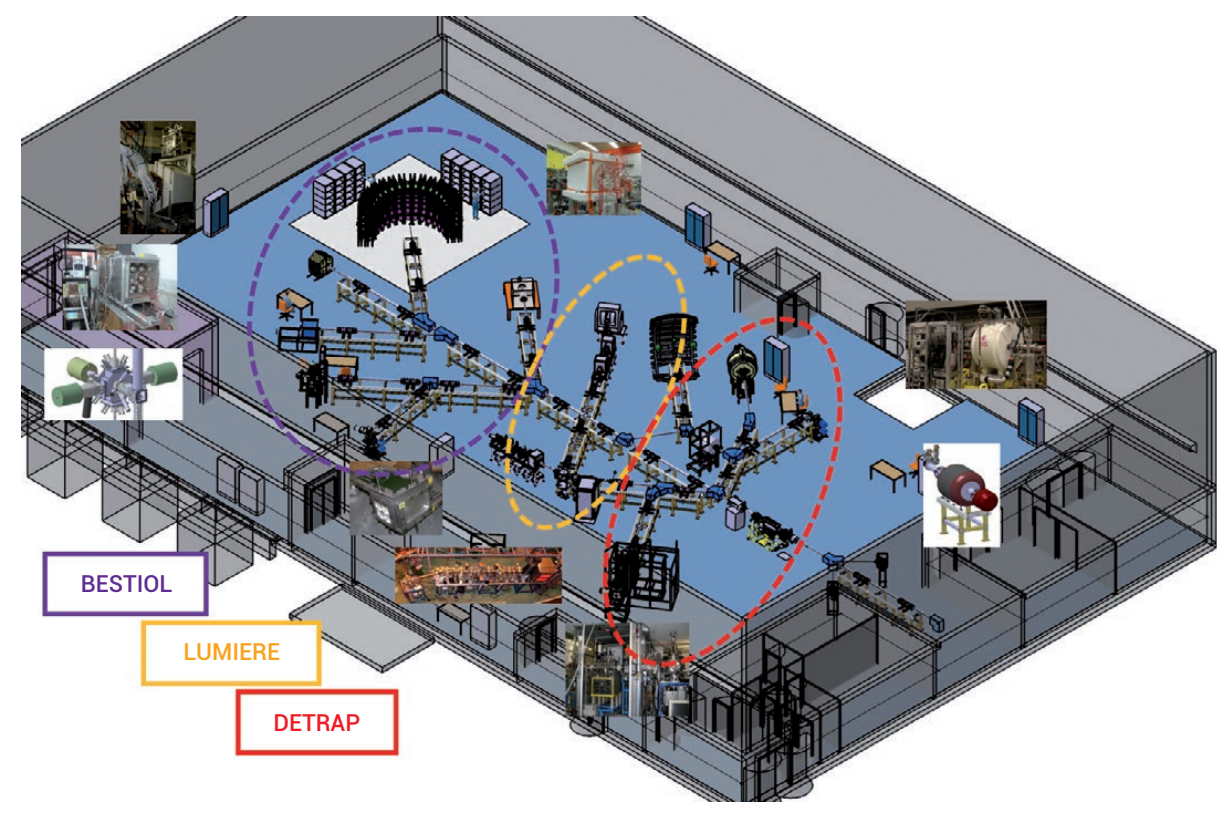

7. Aperçu de la plate-forme expérimentale DESIR. Une arête centrale délivre à un ensemble de dispositifs expérimentaux regroupés en trois sous-ensembles, DETRAP (en rouge), LUMIERE (en jaune) et BESTIOL (en violet), les faisceaux d'ions radioactifs issus de différents sites de production du GANIL. À la grande variété et à la pureté des faisceaux d'ions disponibles s'ajoutent la diversité et la complémentarité des outils de mesures qui seront mis en œuvre pour percer les secrets des noyaux exotiques.

Le projet $\mathrm{S}^{3}$ dans son ensemble est porté par le GANIL et une collaboration de laboratoires français. Il a reçu le soutien de la région (Basse-) Normandie (CPER) et a été sélectionné dans le cadre du premier appel à projet EQUIPEX (ANR-10EQPX-46-01).

\section{La plate-forme DESIR : percer les secrets des noyaux exotiques}

L'installation DESIR [5, 6] (Désintégration, Excitation et Stockage d'Ions Radioactifs) est une plate-forme expérimentale qui mettra à la disposition des chercheurs des faisceaux d'ions radioactifs uniques et des instruments de mesure de grande efficacité et de haute précision.

Le programme des recherches qui seront menées auprès de DESIR porte sur :

- l'évolution de la structure et de la forme du noyau atomique en fonction de son nombre de protons et de neutrons,

- les interactions fondamentales agissant au cour du noyau,

- les modes rares de radioactivité,

- les processus de synthèse des éléments chimiques dans les étoiles.
L'installation DESIR sera alimentée en faisceaux d'ions radioactifs de basse énergie (moins de $60 \mathrm{keV}$ ) issus de trois sites distincts de production d'ions : l'installation SPIRAL1 du GANIL actuel, dans laquelle les ions radioactifs sont produits par fragmentation de noyaux stables par la méthode ISOL ; l'installation $\mathrm{S}^{3}$ couplée au LINAC de SPIRAL2, où les noyaux seront produits dans des réactions de fusion nucléaire ; et, à terme, l'installation de la phase 2 de SPIRAL2 (fig. 2).

Cette complémentarité des modes de production sera renforcée par l'utilisation sur SPIRAL1 et sur la phase 2 de SPIRAL2 de différents types de sources d'ions adaptées aux propriétés chimiques des éléments à ioniser. Le dispositif innovant REGLIS installé sur $\mathrm{S}^{3}$ et décrit plus haut permettra, au moyen de lasers et d'un dispositif de séparation des ions par temps de vol, de sélectionner les ions produits suivant le nombre de protons (Z) et de nucléons (A) que comptent leurs noyaux. Enfin, tous les faisceaux délivrés à l'installation DESIR pourront être séparés efficacement en masse au moyen d'un séparateur de haute résolution. 


\section{$>>$}

Les équipements expérimentaux prévus sur DESIR sont en cours de construction dans différents laboratoires français et étrangers. Ils seront répartis en trois groupes fonctionnels complémentaires, repérés par des couleurs différentes sur la figure 7 : l'ensemble DETRAP regroupe des dispositifs de piégeage d'ions dédiés à leur purification, à la mesure de la masse de leurs noyaux ainsi qu'à l'étude de leurs propriétés de décroissance radioactive. L'installation LUMIERE sera constituée d'une ligne de spectroscopie laser colinéaire et d'un dispositif de polarisation des noyaux par laser : elle permettra d'accéder à la structure et à la forme des noyaux. Enfin, l'ensemble BESTIOL sera constitué de plusieurs détecteurs de particules chargées, de rayonnement gamma et de neutrons. Cet ensemble permettra d'étudier en détail la désintégration des noyaux exotiques délivrés à l'installation DESIR.
Le projet scientifique DESIR est porté par une collaboration forte de plus d'une centaine de personnes issues de laboratoires de recherche français et étrangers. Les faisceaux d'ions et les dispositifs expérimentaux seront également mis à la disposition d'autres communautés scientifiques dans le cadre de recherches pluridisciplinaires. La construction des tunnels permettant d'acheminer les faisceaux d'ions depuis SPIRAL1 et $\mathrm{S}^{3}$, ainsi que celle du hall d'expériences débutera fin 2020. Les premiers faisceaux devraient être disponibles pour la communauté scientifique trois ans plus tard.

La construction de l'infrastructure et de certains des dispositifs expérimentaux est financée par un accord franco-allemand, l'Agence Nationale de la Recherche (programmes EQUIPEX et ANR), et avec le soutien d'universités et de différentes régions.

\section{Bilan et perspectives}

SPIRAL2 est une installation de pointe et complète, dont l'objectif est d'approfondir la recherche en physique nucléaire. Après dix ans d'efforts consacrés à gravir des sommets techniques et réglementaires, les acteurs de l'aventure SPIRAL2 ont pu goûter au premier fruit de leur labeur le 19 décembre 2014 : un faisceau en sortie de source d'ions a été délivré. La seconde étape importante a été l'accélération d'un premier faisceau (de protons) par le RFQ le 3 décembre 2015. La prochaine étape consistera en l'accélération d'un faisceau dans le LINAC, dont l'ensemble des cavités a été mis en froid pour la première fois en novembre 2017. À cette fin, les efforts sont maintenant portés sur la réalisation et les essais des équipements qui garantiront à l'Autorité de Sûreté Nucléaire la sûreté de l'installation. L'autorisation d'exploitation, permettant cette accélération, est attendue fin 2018 .

\section{Notes et références}

1 - Pour une revue plus complète des installations de production d'ions radioactifs existantes ou en projet, voir : Y. Blumenfeld, T. Nilsson et P. Van Duppen, Phys. Scr. T152 (2013) 014023.

2. On peut citer I'IRFU/SACM, I'IRFU/SIS, I'IRFU/SPHN/LENAC du CEA/DRF, I'IPNO, le LPSC, I'IPHC, le LPC Caen, le CENBG du CNRS/IN2P3, ainsi que le GANIL (CEA-IN2P3), et en ce qui concerne l'étranger : I'IFIN-HH (Roumanie), I'INFN-Catane (Italie), I'ALN (USA), BARC (Inde)...

3-X. Ledoux et al., "The Neutrons for Science Facility at SPIRAL-2", Rad. Prot. Dosimetry, 180 (2018) 115-119.

4. C. Stodel, D. Boilley, J. Piot et D. Ackermann,

"À la recherche de l'ultime atome", Reflets de la physique 54 (2017) 14-19.

5• www.cenbg.in2p3.fr/desir/

6. J.-C. Thomas et B. Blank, "The DESIR facility at SPIRAL2", Proceedings of the French-Japanese Symposium on Nuclear Structure Problems, January 5-8, 2011, RIKEN, Japan, World Scientific, ISBN: 978-981-441794-5, p. 224. 